\title{
Immunobiotics mechanisms of action and prospects of use in veterinary medicine.
}

\author{
Aleksandr Refeld ${ }^{1, *}$, Anna Bogdanova ${ }^{1}$, Evgeniya Prazdnova $^{1}$, Alexey Beskopylny ${ }^{2}$, \\ Anastasiya Olshevskaya ${ }^{2}$, Tatyana Maltseva ${ }^{2}$, and Vladislav Zubtsov ${ }^{2}$ \\ ${ }^{1}$ Academy of Biology and Biotechnology of Southern Federal University, 344090, Stachki avenue, \\ 194 building 1, Rostov-on-Don, Rostov obl., Russia \\ ${ }^{2}$ Don State Technical University, 344003, Gagarin sq., 1, Rostov-on-Don, Russia
}

\begin{abstract}
Probiotics are becoming more and more common means of combating intestinal diseases of various origins: infectious pathologies, chronic inflammation, autoimmune disorders. The complex action, coupled with low side effects, makes probiotics promising drugs, especially in veterinary medicine, with an increasing trend towards the inefficient use of antibiotics in the livestock industry. One of the main mechanisms of probiotics action - modulation of host immunity - is perhaps the most difficult and, at the same time, the most actively studied since it is crucial for therapy. Immunobiotics (probiotics that modulate the host's immune response) interact with various innate and adaptive immune cells, changing the expression of pro- and anti-inflammatory cytokines. This action is provided by both the cellular components of probiotic microorganisms and their metabolites and is primarily associated with the host's immunocompetent cells' pattern-recognition receptors, although other molecular mechanisms also exist. This review aims to briefly describe both the molecular mechanisms of immunomodulation by probiotics and the prospects for their use in veterinary medicine.
\end{abstract}

\section{Introduction}

Probiotics are defined as microorganisms that are beneficial to the host if taken in adequate amounts [1]. They are used to treat various diseases, such as autoimmune and infectious mucosal pathologies, inflammatory bowel disease (IBD) [2]. Probiotics capable of modulating the immune response in the host's body are called immunobiotics [6]. Some of the most common genera of microorganisms used as probiotics are Lactobacillus, Bifidobacterium [3]. The mechanisms of action of probiotics are diverse and include reinforcement of epithelial barriers, adhesion to the intestinal mucosa and concomitant inhibition of pathogen adhesion, competitive exclusion of pathogenic microorganisms, production of antimicrobial substances, and modulation of the immune system [4]. Of all

${ }^{*}$ Corresponding author: acalexdc99@gmail.com 
these mechanisms, it is the modulation of the immune response that is the most difficult to study. This is primarily due to the complex structure of eukaryotic immunity and the sophisticated mechanisms of its self-regulation. We will try to briefly draw up a general scheme for immunity modulation by probiotic microorganisms at different levels, with some examples. We will also touch on the prospects for the use of probiotics in veterinary medicine.

\section{Interaction of Probiotics with Host Immunity}

Probiotics are capable of inducing both pro-inflammatory and anti-inflammatory immune responses. Induction of the former can be used to treat infectious and oncological diseases, while the latter is capable of alleviating autoimmune and some infectious diseases [5].

The induction mechanisms lie in interaction with various cells of the host organism, namely with cells of the intestinal epithelium (epithelial cells, $M$ cells), cells of innate (macrophages, dendritic cells, natural killer cells) and adaptive (B-lymphocytes, various subpopulations of T-lymphocytes) immunity. These interactions lead to a change in the cytokine profile and, therefore, to the induction of a pro-inflammatory or anti-inflammatory immune response. This article will also briefly touch on the root causes of alterations in the cytokine profile: effects on signaling pathways and transcription factors. It should be noted that the molecular side of this issue is only beginning to be clarified at the level of signaling pathways. Most of the research is based on analyzing specific cytokines' expression or content in biological material before and after probiotic exposure. The situation is further complicated by the fact that the effects of probiotics on the immune system can vary significantly between normal and pathological conditions.

\subsection{Immunobiotics interactions with intestinal epithelial cells}

Various probiotic strains increase mucin production by epithelial cells, modulate cytokine production through interaction with pattern recognition receptors (PRRs), and affect PRR synthesis (e.g., TLR-2). They are also able to induce prostaglandin synthesis via COX-2 [7]. Interaction with $M$ cells is crucial for the entire immune response since $M$ cells' primary function is to transport antigens from the intestinal lumen directly to the lymphoid tissue (GALT, Peyer's patches), where they will be processed [8]. 


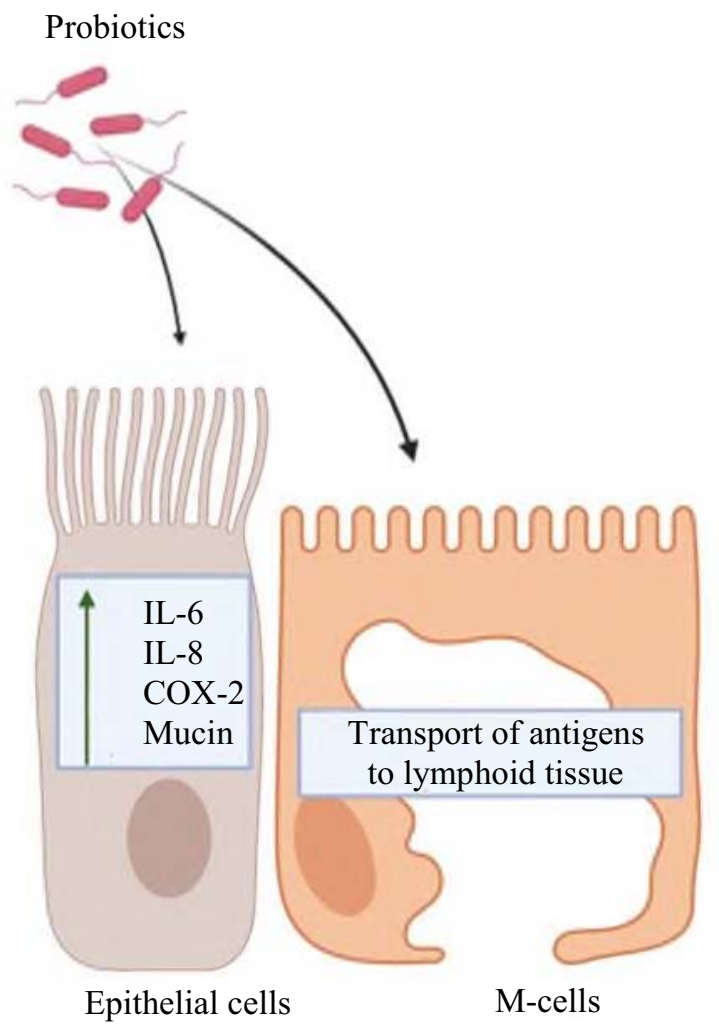

Fig. 1. Immunobiotics interactions with intestinal epithelial cells. IL-6 - pro-inflammatory cytokine; IL-8 - chemokine, neutrophil taxis factor; COX-2 - Cyclooxygenase-2, produces prostaglandins.

\subsection{Immunobiotics interactions with innate immune cells}

\subsubsection{DC}

Lactobacillus rhamnosus and Lactobacillus delbrueckii significantly reduce the expression of surface co-stimulatory signals for $\mathrm{T}$ lymphocytes (CD80, CD83, CD86), proinflammatory IL12, and the transcription factor NF-kB, while increasing the expression of indoleamine-2,3-dioxygenase and IL-10. L. reuteri and L. casei can, in addition to the effects of L. rhamnosus and L. delbrueckii, induce the expression of IFN-gamma and direct the immune response towards both Treg and Th1 [9]. 


\section{Probiotics}
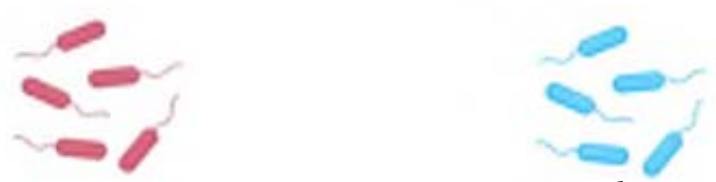

L. reuteri

L. rhamnosus

L. casei

L. delbrueckii

Bifidobacteria

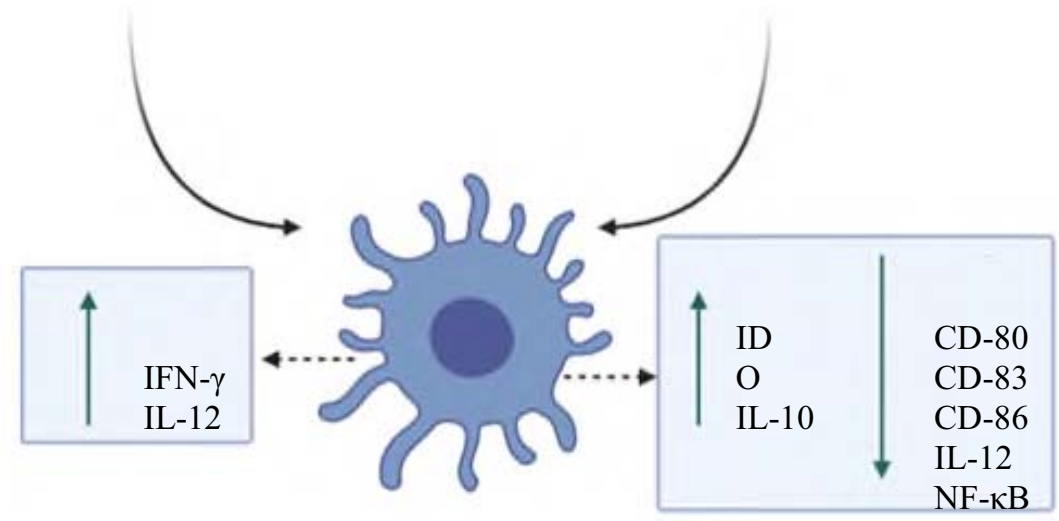

Dendritic cells

(DC)

Fig. 2. Immunobiotics interactions with dendritic cells. Red (left) shows probiotic microorganisms that induce a pro-inflammatory immune response, blue (right) - anti-inflammatory. IFN- $\gamma$ - interferongamma, a pro-inflammatory cytokine; IL-12 - pro-inflammatory cytokine; IDO - indolamine-2,3dioxygenase, oxidizes tryptophan and prevents its conversion into serotonin, an inflammatory mediator; IL-10 - anti-inflammatory cytokine; CD-80, CD-83, CD-86 - co-stimulating signals for effector T-lymphocytes; NF- $\mathrm{KB}$ is a transcription factor that predominantly causes pro-inflammatory reactions.

\subsubsection{Macrophages and monocytes}

L. paracasei DG induces the expression of the pro-inflammatory cytokines TNF- $\alpha$, IL-6, IL-8, and CCL-20 [10]. LAVRI-A1, L. rhamnosus GG, L. acidophilus, and Bifidobacteria induce the secretion of anti-inflammatory cytokines IL4, IL10, and TGF- $\beta$ [13]. TGF- $\beta$ provokes the differentiation of $\mathrm{CD}^{+}{ }^{+} \mathrm{T}$-lymphocytes into Treg/Th17, and the effect of IL-4 is to differentiate the same $\mathrm{CD} 4^{+} \mathrm{T}$-lymphocytes into $\mathrm{Th} 2$ cells.

The effect on natural killer cells is mainly carried out by modulating the secretion of cytokines by other cells. Thus, IL-12 is their natural activator, and IL-10 is an inhibitor. 


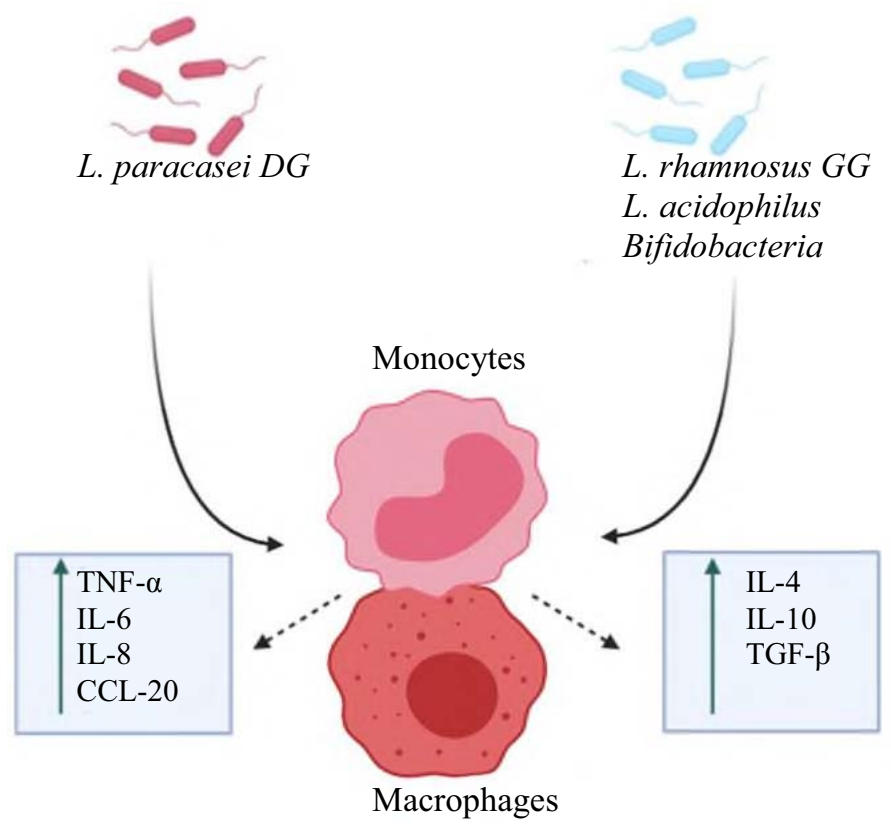

Fig. 3. Immunobiotics interactions with macrophages and monocytes. Red (left) shows probiotic microorganisms that induce a pro-inflammatory immune response, blue (right) - anti-inflammatory. TNF- $\alpha$, IL-6 - pro-inflammatory cytokines; IL-8 - chemokine, neutrophil taxis factor; CCL-20 chemokine, lymphocyte taxis factor; IL-4, IL-10, TGF- $\beta$ - anti-inflammatory cytokines.

\subsection{Immunobiotics interactions with adaptive immune cells}

It is carried out mainly through the influence on the secretion of cytokines by cells of innate immunity. Nevertheless, we will give several examples of both the indirect and direct influence of probiotics on immunity's adaptive link.

\subsubsection{Treg}

L. Acidophilus L-92 and Bifidobacteria strains significantly reduce the concentration of IgE and IFN- $\gamma$ in blood plasma and induces the production of TGF- $\beta$ in Peyer's patches. TGF- $\beta$ is an essential factor for $\mathrm{CD}^{+}$T-lymphocytes differentiation into Treg cells [11]. Bifidobacteria are able to reduce the severity of the allergic Th2 immune response. Furthermore, they increase the number of Tregs and IL-10-positive cells [12].

\subsubsection{Th17}

Various Lactobacillus strains significantly reduce the level of expression of IL-17, the main effector pro-inflammatory cytokine of Th17 cells, thereby reducing the pro-inflammatory immune response [14].

Th1 activation occurs predominantly by IFN- $\gamma$ and IL-12, while Th2 is activated by the anti-inflammatory cytokine IL-4.

\subsubsection{B-cells}


Many probiotics promote the production of $\operatorname{IgA}$ antibodies, which act primarily in the mucosa and support its barrier function. At the same time, probiotics reduce the production of IgE antibodies associated with allergic reactions [15].

\section{Pattern recognition receptors and signaling pathways}

This multifaceted action of probiotics is most likely associated with the action of parts of microorganisms on various PRR (pattern-recognition receptors), such as NLR (NOD-like receptors) and TLR (Toll-like receptors).

The best-studied TLRs in the context of probiotics are TLR4, which induces a proinflammatory response, and TLR2 and TLR9, which are mainly responsible for the antiinflammatory response.

Probiotic microorganisms (mostly gram-positive Lactobacillus and Bifidobacterium) block the interaction of lipopolysaccharide (a component of the cell wall of gram-negative bacteria) with its receptor - the TLR4\CD14 complex. This leads to the inactivation of the pro-inflammatory transcription factor NF-kB [16]. This effect is achieved due to the attachment of lipoteichoic acid (a component of the cell wall of gram-positive bacteria) to its receptor - TLR2.

Suppression of activity is observed not only for NF-kB but also for various MAP kinases, including p38 [17, 18]. However, some lactobacilli are able to activate TLR4 due to the small number of LPS in their cell walls, as well as with the help of cytoplasmic heat shock proteins. Furthermore, the TLR4-dependent response induced by Lactobacillus is crucial in Salmonella enterica infection [19].

TLR9 can act as both an activator and an inhibitor of the NF-kB pathway in polarized epithelial cells. Binding of bacterial DNA to the TLR9 receptor on the basolateral surface of cells induces degradation of IkB- $\alpha$ and activation of NF-kB, and on the apical surface to the accumulation of unutilized cytoplasmatic IkB and inhibition of NF-kB [20]. It is interesting to note that the TLR9 ligand is not just bacterial DNA, but its CpG islands. Moreover, since Lactobacillus species differ significantly in their $\mathrm{C}+\mathrm{G}$ composition, the effects of binding to TLR9 may differ from species to species [21].

There is little data on the interaction of probiotics with NLRs. L. delbrueckii subsp. bulgaricus NIAI B6 and L. gasseri JCM1131 can increase the expression of NLRP3 in the GALT [22]. The same authors found that this receptor can interact with the ligands of the TLR2 and TLR9 receptors, providing not anti-inflammatory but pro-inflammatory action (activation of caspase-1, which is necessary for the maturation of IL-1 from the precursor). 


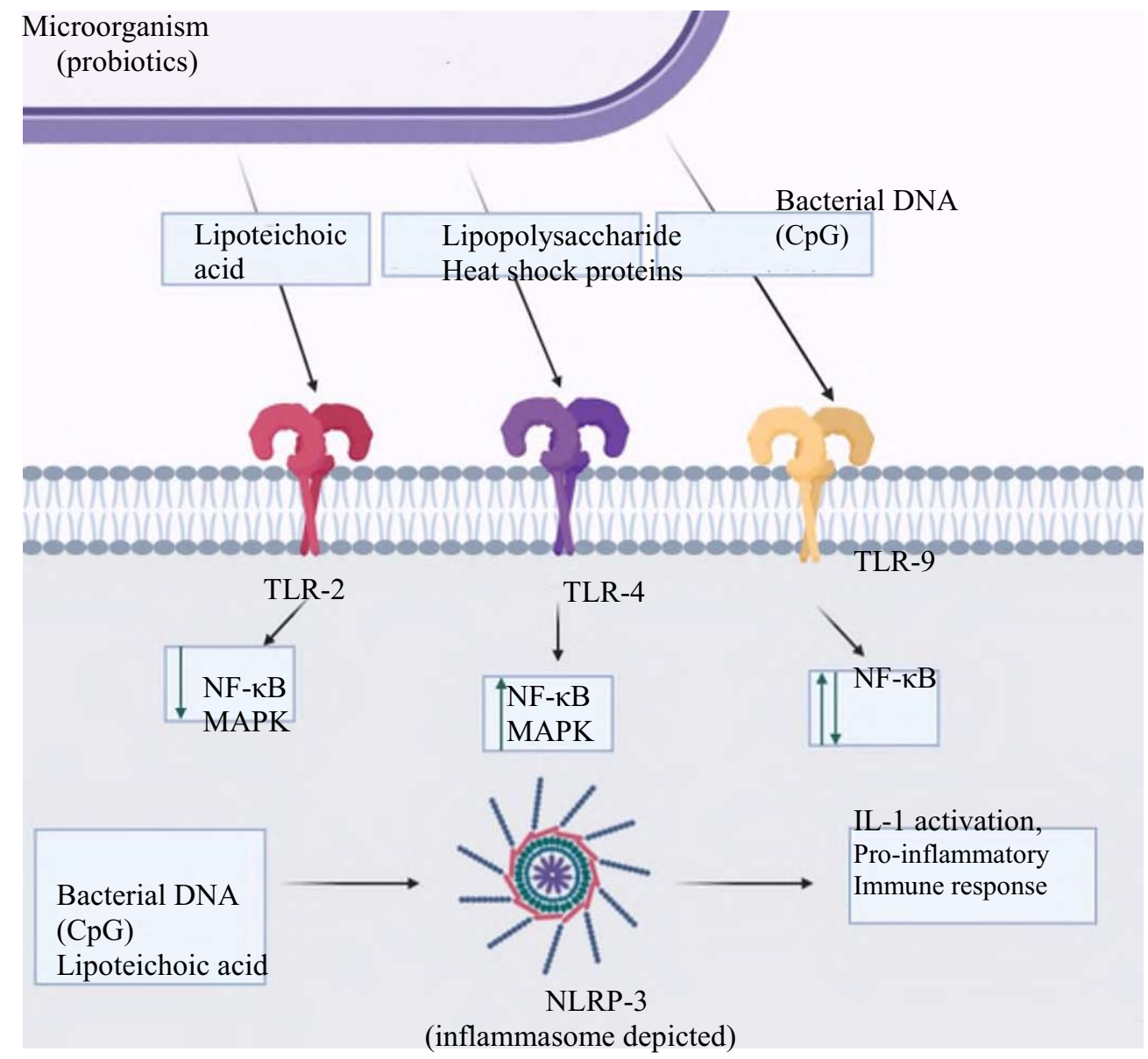

Fig. 4. PRR affected by probiotic bacteria. NF- $\kappa \mathrm{B}$ - a transcription factor that predominantly causes pro-inflammatory reactions; MAPK - MAP kinases associated (especially p38) predominantly with the pro-inflammatory immune response; IL-1 - pro-inflammatory cytokine.

\section{Immunomodulating effect of probiotic bacteria metabolites}

\subsection{Short-chain fatty acids}

Butyrate is capable of both initiating Treg differentiation [23] and stimulating the production of IL-8, a pro-inflammatory cytokine [24]. It has also been shown that butyrate is able to enhance the production of MUC2 mucin. It is possible that butyric acid performs its functions at the epigenetic level since it is a natural inhibitor of histone deacetylase [25].

Lactic acid can reduce the proliferation of cytotoxic T-lymphocytes, and with it, the expression of pro-inflammatory cytokines IFN- $\gamma$ and IL-2 [26]. It also promotes the transformation of pro-inflammatory M1 macrophages into regenerative M2 macrophages [27]. 
Hydroxyisocaproic acid (HICA) reduces the production of pro-inflammatory cytokines, IL-1 $\beta$ and TNF- $\alpha$, and inflammation-associated metalloproteinase- 9 and myeloperoxidase [28].

\subsection{Peptides and proteins}

Protein p40, isolated from Lactobacillus rhamnosus GG, reduces the production of TNF, IL-6, keratinocyte chemoattractant (KC), and IFN- $\gamma$ and thereby ameliorates intestinal inflammation through the activation of EGFR (epidermal growth factor receptor) [29].

Bacteriocins, nisin in particular, also show immunomodulatory properties: in mouse studies, it induces an immune boost by increasing the production of IL- 6 and IL- 10 . Although nisin does not inhibit the functional capacity of leukocytes concerning the production of IL-1 $\beta$ and IL-6, in high doses it is cytotoxic [30].

\section{Possibilities of using immunobiotics in veterinary medicine}

Given the many proven effects of probiotics on mammals and birds' immune systems, it makes sense to use them in veterinary medicine to treat infectious diseases (especially in conditions of increasing antibiotic resistance) and immune disorders.

Early studies indicate that probiotics Bifidobacterium pseudolongum M-602 or Lactobacillus acidophilus LAC-300 reduced the incidence of infectious diarrhea in calves [31]. Chicken studies indicate that Lactobacillus salivarius, L. johnsonii, L. reuteri, L. crispatus, and L. gasseri may be involved in bird defense against the H9N2 avian influenza virus. The use of immunobiotics against viral diseases of farm animals is the subject of many studies, the results of which can be called promising [32,33]. Studies in rat models of vaginal candidiasis have shown Candida growth inhibition by $60-70 \%$ with intravaginal administration of probiotic Lactobacillus strains [34]. Bifidobacterium infantis prevents infection with Salmonella typhimurium and pathogenic Escherichia coli in mice through the production of acetic acid [35].

Lactobacillus jensenii TL2937 maintains and improves intestinal homeostasis in piglets. This probiotic significantly reduced the concentration of the complement system components and C-reactive protein in the blood and improved the productivity and quality of animal carcass in general [36]. Mouse models have also shown a reduced severity of chronic colitis when exposed to the bacteria of the genus Clostridium [37]. Genetically modified Lactococcus lactis and other lactobacilli synthesizing IL-10 significantly reduce inflammatory bowel disease manifestations in various mammalian models [38]. Many studies have also shown a positive effect of immunobiotics on the productivity of broiler chicks and their stimulation of intestinal immunity in response to the pathogenic microorganisms.

Moreover, immunobiotics are seriously considered as an alternative to antibiotics in the poultry industry [39]. Studies in dogs show that early use of Lactobacillus rhamnosus GG (LGG) significantly reduces allergen-specific IgE and prevents allergic diseases in the long term [40]. Allergy symptom relief using probiotic Lactobacillus rhamnosus HN001 has been shown in pigs [41]. The use of the multi-species probiotic GI7 reduces skin lesions associated with atopic dermatitis in mice. This result is probably associated with the induction of the generation of T-regulatory cells [42]. Also, in many studies on mammalian cell cultures and model organisms, it has been shown that a large number of Lactobacillus strains are capable of inhibiting the growth of cervical cancer cells [43].

\section{Summary}


Immunobiotics can have a systemic effect on the state of the intestinal immune system. This action is probably carried out due to the interaction of prokaryotic cell components with pattern-recognition receptors of the host's immune cells. However, metabolites like short-chain fatty acids and peptides secreted by these organisms also play an important role and may involve other mechanisms of influence on the immune system (for example, butyrate is a histone deacetylase inhibitor and could participate in epigenetic regulation). The effect of immunobiotics on the immune system can result in both a pro-inflammatory and anti-inflammatory immune response, and the choice of the preferred pathway will be determined by the genus, species, and even the strain of the microorganism used as a probiotic, as well as by the state of the immune system and the general state of health of the host. The presence of autoimmune, chronic inflammatory, allergic and oncological diseases, as well as the presence of pathogenic microorganisms in the intestine of the host, can affect the action of probiotics. Therefore, their application in clinical practice requires a lot of preliminary studies and careful standardization. However, the use of probiotics in veterinary medicine is already an effective approach for preventing infectious diseases, especially in the context of developing antibiotic resistance. Despite the fact that probiotics have shown some efficacy when used against autoimmune and allergic disorders and cancer, their use in this area is seen as a more distant prospect due to the aforenamed problems.

The research was financially supported by the Ministry of Science and Higher Education of the Russian Federation within the framework of the state task in the field of scientific activity (Southern Federal University, no. 0852-2020-0029) and by the Government of the Russian Federation (contract No. 075-15-2019-1880)

Authors acknowledge the support of the Government of the Russian Federation (contract No. 075-152019-1880).

\section{References}

1. N. T. Williams, "Probiotics." American Journal of Health-System Pharmacy, 67(6), 449-458 (2010)

2. K. G. Rout, et al., "Benefaction of probiotics for human health: A review." Journal of food and drug analysis, 26(3), 927-939 (2018)

3. A. N. Vlasova, et al., "Comparison of probiotic lactobacilli and bifidobacteria effects, immune responses and rotavirus vaccines and infection in different host species." Veterinary immunology and immunopathology, 172, 72-84 (2016)

4. P. Markowiak, K. Śliżewska, "Effects of probiotics, prebiotics, and synbiotics on human health." Nutrients, 9(9), 1021 (2017)

5. H.-J. Kang, S.-H. Im. "Probiotics as an immune modulator." Journal of nutritional science and vitaminology, Supplement, 61, 103-105 (2015)

6. J. Villena, K. Haruki, "The modulation of mucosal antiviral immunity by Immunobiotics: could they offer any benefit in the SARS-CoV-2 pandemic?." Frontiers in Physiology, 11 (2020)

7. L. Khailova, et al., "Bifidobacterium bifidum reduces apoptosis in the intestinal epithelium in necrotizing enterocolitis." American Journal of PhysiologyGastrointestinal and Liver Physiology, 299(5), 1118-1127 (2010)

8. N. Saad, et al., "An overview of the last advances in probiotic and prebiotic field." LWT-Food Science and Technology, 50(1), 1-16 (2013) 
9. S.-A. Esmaeili, et al., "Generation of tolerogenic dendritic cells using Lactobacillus rhamnosus and Lactobacillus delbrueckii as tolerogenic probiotics." Journal of cellular biochemistry, 119(9), 7865-7872 (2018)

10. S. Balzaretti, et al., "A novel rhamnose-rich hetero-exopolysaccharide isolated from Lactobacillus paracasei DG activates THP-1 human monocytic cells." Applied and environmental microbiology, 83(3) (2017)

11. K. Honda, R. L. Dan, "The microbiome in infectious disease and inflammation." Annual review of immunology, 30, 759-795 (2012)

12. L.-L. Zhang, et al., "Oral Bifidobacterium modulates intestinal immune inflammation in mice with food allergy." Journal of gastroenterology and hepatology, 25(5), 928-934 (2010)

13. O. N. Donkor, et al. "Cytokine profile and induction of T helper type 17 and regulatory T cells by human peripheral mononuclear cells after microbial exposure." Clinical \& Experimental Immunology, 167(2), 282-295 (2012)

14. H.-K. Kwon, et al., "Generation of regulatory dendritic cells and CD4+Foxp3+T cells by probiotics administration suppresses immune disorders." Proceedings of the National Academy of Sciences, 107(5), 2159-2164 (2010)

15. Md. Azad, et al., "Immunomodulatory effects of probiotics on cytokine profiles." BioMed Research International 2018 (2018).

16. H. P. Mykhal'chyshyn, P. M. Bodnar, N. M. Kobyliak, "Effect of probiotics on proinflammatory cytokines level in patients with type 2 diabetes and nonalcoholic fatty liver disease." Likars' ka sprava, 2, 56 (2013)

17. C. M. Carey, K. Magdalena, "Lactic acid bacteria and bifidobacteria attenuate the proinflammatory response in intestinal epithelial cells induced by Salmonella enterica serovar Typhimurium." Canadian Journal of Microbiology, 59(1), 9-17 (2013)

18. L. Giahi, et al., "Regulation of TLR4, p38 MAPkinase, I $\mathrm{KB}$ and miRNAs by inactivated strains of lactobacilli in human dendritic cells." Beneficial microbes, 3(2), 91-98 (2012)

19. N. A. Castillo, P. Gabriela, de M. de LeB. Alejandra, "Oral administration of a probiotic Lactobacillus modulates cytokine production and TLR expression improving the immune response against Salmonella enterica serovar Typhimurium infection in mice." BMC microbiology, 11(1), 177 (2011)

20. M. T. Abreu, "Toll-like receptor signalling in the intestinal epithelium: how bacterial recognition shapes intestinal function." Nature Reviews Immunology, 10(2), 131-144 (2010)

21. J. M. Wells, "Immunomodulatory mechanisms of lactobacilli." Microbial cell factories, 10(1) (BioMed Central, 2011)

22. M. Tohno, et al., "Immunobiotic Lactobacillus strains augment NLRP3 expression in newborn and adult porcine gut-associated lymphoid tissues." Veterinary immunology and immunopathology, 144(3-4), 410-416 (2011)

23. N. Singh, et al., "Activation of Gpr109a, receptor for niacin and the commensal metabolite butyrate, suppresses colonic inflammation and carcinogenesis." Immunity, 40(1), 128-139 (2014)

24. A. Murzyn, et al., "The effect of Saccharomyces boulardii on Candida albicansinfected human intestinal cell lines Caco-2 and Intestin 407." FEMS microbiology letters, 310(1), 17-23 (2010) 
25. R. B. Canani, et al., "Potential beneficial effects of butyrate in intestinal and extraintestinal diseases." World journal of gastroenterology: WJG, 17(12), 1519 (2011)

26. F. Hirschhaeuser, G. A. S. Ulrike, M.-K. Wolfgang, "Lactate: a metabolic key player in cancer." Cancer research, 71(22), 6921-6925 (2011)

27. O. R. Colegio, et al. "Functional polarization of tumour-associated macrophages by tumour-derived lactic acid." Nature, 513(7519), 559-563 (2014)

28. M. T. Nieminen, et al., "DL-2-hydroxyisocaproic acid attenuates inflammatory responses in a murine Candida albicans biofilm model." Clinical and Vaccine Immunology, 21(9), 1240-1245 (2014)

29. F. Yan, et al., "Colon-specific delivery of a probiotic-derived soluble protein ameliorates intestinal inflammation in mice through an EGFR-dependent mechanism." The Journal of clinical investigation, 121(6), 2242-2253 (2011)

30. S. K. Tiwari, et al., "Probiotics at war against viruses: What is missing from the picture?." Frontiers in Microbiology, 11, 1877 (2020)

31. J. Villena, et al., "Immunobiotics for the bovine host: their interaction with intestinal epithelial cells and their effect on antiviral immunity." Frontiers in immunology, 9, 326 (2018)

32. J. Villena, et al., "Immunobiotics for the bovine host: their interaction with intestinal epithelial cells and their effect on antiviral immunity." Frontiers in immunology, 9, 326 (2018)

33. V. Julio, M. G. Vizoso-Pinto, H. Kitazawa, "Intestinal innate antiviral immunity and immunobiotics: beneficial effects against rotavirus infection." Frontiers in immunology, 7, 563 (2016)

34. L. Ting, et al., "Local probiotic lactobacillus crispatus and lactobacillus delbrueckii exhibit strong antifungal effects against vulvovaginal candidiasis in a rat model." Frontiers in Microbiology, 10, 1033 (2019)

35. S. Fukuda, et al., "Bifidobacteria can protect from enteropathogenic infection through production of acetate." Nature, 469(7331), 543-547 (2011)

36. Y. Suda, et al., "Immunobiotic Lactobacillus jensenii as immune-health promoting factor to improve growth performance and productivity in post-weaning pigs." BMC immunology, 15(1), 1-18 (2014)

37. T. Chiba, S. Hiroshi, "Indigenous clostridium species regulate systemic immune responses by induction of colonic regulatory T cells." Gastroenterology, 141(3), 1114$1116(2011)$

38. S. Shigemori, S. Takeshi, "Applications of genetically modified immunobiotics with high immunoregulatory capacity for treatment of inflammatory bowel diseases." Frontiers in immunology, 8, 22 (2017)

39. A. A. Khaskheli, et al., "A review on the influence of dietary immunobiotics on the performance, intestinal morphology and immune-related gene expression in posthatched broiler chicks." Aceh Journal of Animal Science, 5(1), 57-67 (2020)

40. R. Marsella, S. Domenico, A. Kim, "Early exposure to probiotics in a canine model of atopic dermatitis has long-term clinical and immunological effects." Veterinary immunology and immunopathology, 146(2), 185-189 (2012)

41. D. J. Thomas, et al., "Lactobacillus rhamnosus HN001 attenuates allergy development in a pig model." PLoS One, 6(2), e16577 (2011) 
42. J.-H. Shin, C. Myung-Jun, S. Jae-Gu, "A multistrain probiotic formulation attenuates skin symptoms of atopic dermatitis in a mouse model through the generation of CD4+ Foxp3+ T cells." Food \& nutrition research, 60(1), 32550 (2016)

43. X. Yang, et al., "Role of Lactobacillus in cervical cancer." Cancer Management and Research, 10, 1219 (2018)

44. M. Mazanko, E. Prazdnova, D. Rudoy, A. Ermakov, A. Olshevskaya, T. Maltseva, E3S $W e b$ of Conferences, 175, 01010 (2020) INTERAGROMASH 2020 https://doi.org/10.1051/e3sconf/202017501010 